\title{
Genetic Polymorphisms at TNF Microsatellites from the HLA CLASS III Region in a South Tunisian Population
}

\author{
Nadia Mahfoudh*, Arwa Kamoun, Lilia Gaddour, Faiza Hakim, Hafedh Makni \\ Immunology Department, Service De Laboratoire, University Hospital of Hedi Chaker, Sfax, Tunisia \\ Email: $\underline{\text { Lahiani@voila.fr }}$
}

Received 9 July 2014; revised 19 August 2014; accepted 23 September 2014

Copyright (C) 2014 by authors and OALib.

This work is licensed under the Creative Commons Attribution International License (CC BY).

http://creativecommons.org/licenses/by/4.0/

(c) ()

\section{Abstract}

To evaluate the tumor necrosis factor (TNF) a-b-c microsatellite polymorphism in South Tunisia, we studied 123 healthy unrelated controls from South Tunisia. TNF microsatellite alleles were typed using genomic DNA amplified with specific primers. Statistical analyses were performed using ARLEQUIN software. The TNFa6 (18.9\%), TNFa2 (16.4\%), TNFb5 (41.9\%), and TNFc1 (68.7\%) were the most frequent alleles in our population, whereas the TNFa8 and TNFb2 were the least frequent alleles. To investigate the association among the three microsatellites, a detailed haplotype analysis was performed. 236 different haplotypes were possible but there was only 15 haplotypes had frequencies greater than $2 \%$. In our population the most frequent haplotype TNFcab were "TNFc1a6b5" (10.03\%). These results may be informative for studies of the associations of individual TNF region markers with secretion levels, immunity, and disease.

\section{Keywords}

TNF, Microsatellite, Haplotype, Population

Subject Areas: Genetics, Immunology

\section{Introduction}

One group of microsatellites in the HLA class III region is TNF a, b and c. The TNF a and b microsatellites are located 3.5 kilobases upstream of the Lymphotoxin- $\alpha$ (LTA) gene (Figure 1).

The three microsatellite regions span around the TNF region, exhibiting 13 alleles for TNFa (a1-a13), 8 for TNFb (b0-b7), 2 for TNFc (c1-c2).

${ }^{*}$ Corresponding author.

How to cite this paper: Mahfoudh, N., Kamoun, A., Gaddour, L., Hakim, F. and Makni, H. (2014) Genetic Polymorphisms at TNF Microsatellites from the HLA CLASS III Region in a South Tunisian Population. Open Access Library Journal, 1: e929. http://dx.doi.org/10.4236/oalib.1100929 


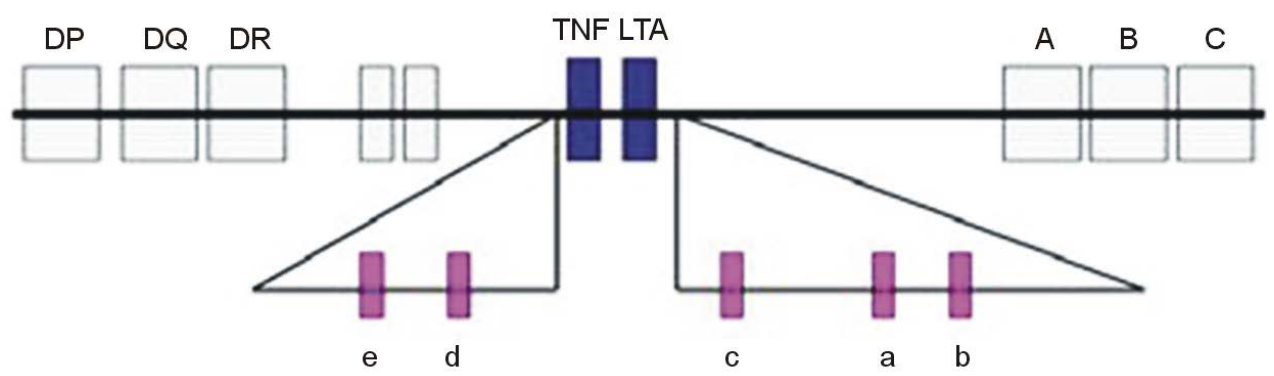

Figure 1. Location and order of 5 TNF microsatellites within the HLA complex on chromosome 6 [1].

TNFa and TNFb are composed of (GT)n and (GA)n repeats, respectively.

The TNFc microsatellite is a (GA)n repeat located within the first intron of the TNF $\alpha$ gene [2].

Hence, the present study is carried out in a Tunisian population to investigate the frequency of these TNF microsatellite alleles and their relationships with five Europeans populations (French, Basque, Greek, Danish and Italian) and Asian Orientals populations (Indian, Japanese and Chinese).

\section{Methods}

1) Sample population

The study was accomplished on a sample of 123 healthy unrelated from south Tunisia. The population sample is the same as the one tested for HLA in the paper published in Ann. Hum. Biol. 2013, 40, 41-47.

The samples of population studied were from different regions of south Tunisia.

2) Microsatellites genotyping

PCR amplification of the polymorphic TNFa, b and c microsatellite was carried out with primers.The forward primer was labeled at the 5'-end with the fluorescent VIC and Pet reagent respectively TNFa, TNFb and TNFc. Table 1 (http://www.ncbi.nlm.nih.gov/gv/mhc/xslcgi.cgi?cmd=mssearch) [3].

To determine the number of the repeat in the region of the TNF gene, the amplified products were denatured for 3 min at $96^{\circ} \mathrm{C}$, mixed with formamide and electrophoresed in an automated DNA sequencer (Applied Biosystems model 310 sequencer). The number of microsatellite repeat was estimated automatically using the GENESCAN 3.1 software (Applied Biosystems) with a size standard (Applied Biosystems). The different alleles of the TNFa microsatellites correspond to fragment sizes as follows in Table 2.

\section{Statistics}

Allele frequencies were estimated by direct counting.

Hardy-Weinberg equilibrium, haplotype frequencies, linkage disequilibrium and their significance as determined by chi 2 test were calculated using the Arlequin version 3.0.

Some statistical parameters of forensic interestsuch as heterozygosity (Hz), power of discrimination (PD), mean exclusion chance (MEC) and polymorphic information content (PIC) were calculated using the software package Power Stats (Promega).

A linkage disequilibrium study was performed using an online software SHEsis (http://202.120.7.14/analysis/myAnalysis.php) [4].

Two LD coefficients, Lewontin's D' and Hill's $\mathrm{r}^{2}$ were evaluated by the same software.

\section{Results}

1) TNF microsatellites alleles

Allele frequencies were shown in Table 3.

A total of 13 alleles at TNFa, 8 alleles at TNFb, and 2 alleles at TNFc microsatellite were observed among South Tunisian population.

For TNFa microsatellite locus PIC values around 0.87 have been observed. 
Table 1. Characteristics of TNF microsatellites.

\begin{tabular}{cc}
\hline Microsatellites & Primer \\
\hline TNFa & F: VIC 5'GCCTCTAGATTTCATCCAGCCACA3' \\
& R: 5'CCTCTCCCCCTGCAACACACA3' \\
TNFb & F: VIC 5'GCACTCCAGCCTAGGCCACAGA3' \\
& R: 5'tgtgtgttgcaggggagagag3' \\
TNFC & F: PET 5'gggaggtctgtcttccgcco3' \\
& R: 5'cgttcaggtggtgtcatggg3' \\
\hline
\end{tabular}

VIC: fluorescent label; PET: fluorescent label.

Table 2. The corresponding length en pb and allele of TNF a, b, c STR.

\begin{tabular}{cccc}
\hline TNF allele & Length en $\mathrm{pb}$ & TNF allele & Length en pb \\
\hline a1 & 98 & b0 & 116 \\
a2 & 100 & b1 & 118 \\
a3 & 102 & b2 & 122 \\
a4 & 104 & b3 & 124 \\
a5 & 106 & b4 & 126 \\
a6 & 108 & b5 & 128 \\
a7 & 110 & b6 & 130 \\
a8 & 112 & B7 & \\
a9 & 114 & & 97 \\
a10 & 116 & & 99 \\
a11 & 118 & c1 & c2 \\
a12 & 120 & 122 & \\
a13 & & & 9 \\
\hline
\end{tabular}

Table 3. Allele frequencies of TNF microsatellite markers in controls.

\begin{tabular}{|c|c|c|c|}
\hline \multirow{2}{*}{ Allele Number } & \multicolumn{3}{|c|}{$\begin{array}{l}\text { Allele frequency } \\
\text { (\%) }\end{array}$} \\
\hline & $\begin{array}{c}\text { TNFa } \\
(\mathrm{n}=246)\end{array}$ & $\begin{array}{c}\text { TNFb } \\
(\mathrm{n}=246)\end{array}$ & $\begin{array}{c}\text { TNFC } \\
(\mathrm{n}=246)\end{array}$ \\
\hline 0 & - & 1.20 & - \\
\hline 1 & 5.30 & 11.40 & 68.70 \\
\hline 2 & 16.40 & 1.60 & 31.30 \\
\hline 3 & 1.60 & 13.80 & \\
\hline 4 & 9.40 & 20.70 & \\
\hline 5 & 12.70 & 41.90 & \\
\hline 6 & 18.90 & 2.80 & \\
\hline 7 & 9.40 & 6.50 & \\
\hline 8 & 0.40 & & \\
\hline 9 & 3.70 & & \\
\hline 10 & 11.10 & & \\
\hline 11 & 4.90 & & \\
\hline 12 & 3.70 & & \\
\hline 13 & 2.80 & & \\
\hline Но & 0.813 & 0.577 & 0.447 \\
\hline PIC & 0.870 & 0.890 & 0.340 \\
\hline $\mathrm{PD}$ & 0.968 & 0.710 & 0.577 \\
\hline
\end{tabular}

n, number of chromosomes; Ho, observed heterozygosity; PIC, polymorphic information content; PD, power of discrimination. 
Likewise, PIC oscillated from 0.34 at TNFc to 0.87 at TNFa, whereas PD varied from 0.57 at TNFc to 0.96 at TNFa. The heterozygosity level for TNFa was particularly high (0.81), and greater than 0.57 for TNFb and 0.44 for TNFc loci.

The present study with a South Tunisian population shows that among TNFa alleles, TNFa6 allele was the most frequent (18.9\%) and TNFa2 (16.4\%) is the second most frequent allele. However TNFa8 had a low frequency in our population ( $0.4 \%)$.

Among TNFb alleles, TNFb4 and b5 were the most frequent (20.7\%, 41.9\% respectively), whereas TNFb0, TNFb2 and TNFb6 were very low in our population (1.2\%, 1.6\%, 2.8\% respectively).

For TNFc, the most frequent allele was TNFc1 (68.7\%).

2) TNF microsatellites Haplotypes

To investigate the association among the three microsatellites, a detailed haplotype analysis was performed in all samples.

Given the observed polymorphism at TNFa, b, and c microsatellites in our population (13, 7, 2 alleles, respectively), 236 different haplotypes were possible but there were only 69 haplotypes observed in our population.

The 15 most frequent haplotypes with frequency greater than 2\% were shown in Table 4.

In our population the most frequent haplotypes TNFc, a, b were TNFc1a6b5 (10\%), TNFc1a10b4 (5.6\%). Two rare haplotypes with frequencies lower than $0.1 \%$ were observed: TNFc2a12b3 and TNFc2a3b1.

Regarding linkage disequilibrium (LD) between microsatellite markers (TNFa, b and c), our results showed a weak LD between TNFa and TNFC ( $\left.\mathrm{D}^{\prime}=0.263, \mathrm{r}^{2}=0.007\right)$, TNFa and TNFb ( $\left.\mathrm{D}^{\prime}=0.424, \mathrm{r}^{2}=0.02\right)$, TNFb and TNFC (D' $=0.328, r^{2}=0.03$ ) (Figure 2).

\section{Discussion}

In a Tunisian study assessing TNFa association with Graves disease [5], 14 alleles were typed in the Tunisian Healthy controls and TNFa2 was the most frequent allele (19.26\%) followed by TNFa7 (14.53\%) and TNFa6 (13.85\%).

Comparing our findings with the studies done on other populations, it was observed that alleles' distribution in our population was different from Europeans [6].

The most common TNFa microsatellite in the European population was TNFa2 with a frequency varying between $20.8 \%$ in the French sample and 32\% in a Danish study [7].

\begin{tabular}{lc} 
Table 4. Haplotype frequency distribution in samples. \\
\hline Haplotype TNFcab & Frequency (\%) \\
\hline c1a6b5 & 10.03 \\
c1a10b4 & 5.64 \\
c1a2b3 & 5.58 \\
c2a2b1 & 5.03 \\
c1a5b5 & 4.35 \\
c1a5b7 & 3.66 \\
c1a6b4 & 3.64 \\
c1a7b5 & 3.03 \\
c1a4b7 & 2.85 \\
c1a12b5 & 2.73 \\
c1a4b5 & 2.54 \\
c1a10b5 & 2.38 \\
c2a4b5 & 2.17 \\
c2a7b1 & 2.10 \\
c2a11b5 & 2.08 \\
\hline
\end{tabular}




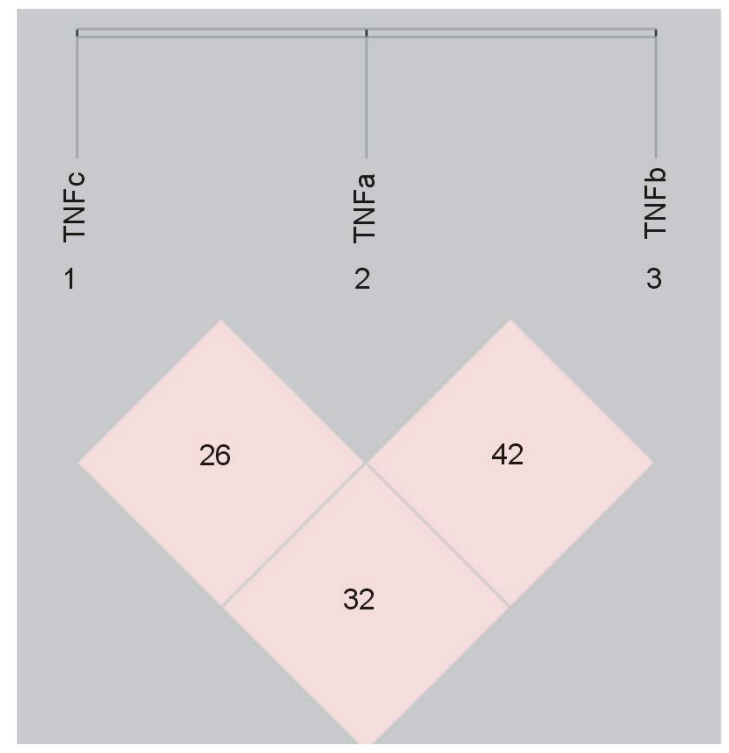

Figure 2. Pairwaise linkage disequilibrium (LD) analysis of TNFc-a-b loci.

However our study placed TNFa2 by itself as the second most represented type (16.4\%), preceded only by TNFa6 (18.9\%).

Comparing our findings with the studies done on Asian Orientals, a different pattern of allele's distribution was observed: In the Japanese population, TNFa13 was the most frequent allele; In Indians and in Chinese, the most frequent TNFa alleles were TNFa10 and TNFa6.

TNFa1 distribution differed among populations. In Basques, it was relatively frequent (11.6\%) but low in the French population as well as in our study (3.1\%, 5.3\% respectively), very low in Danish samples (0.6\%), and nonexistent in the Greeks.

Among TNFb alleles, TNFb5 was the most frequent (41.6\%), whereas TNFb2 and TNFb6 were very low in our study. This pattern of TNFb allele distribution was quite similar to that in the Basque, Danish and Chinese populations [8] but quite different from the French population where TNFb5 was found to have a low frequency (6.7\%).

Analysis of TNFc polymorphism revealed that the observed frequency of TNFc1 allele in our population $(68.7 \%)$ was quite similar to those found in Caucasoid originating from Germany (70.6\%) and from France (77.1\%).

Haplotype studies were powerful than gene frequency studies and can be a useful tool to characterize populations. The most frequent TNFcab haplotype was TNFc1a6b5 (10\%) in our study, TNFc1a12b4 in French (7.3\%) and TNFc1a4b5 in Danish (4.6\%).

These data suggest that the TNF microsatellite haplotypes constitute a highly polymorphic system and that will provide useful information on the association between the TNF marker and the immune diseases.

\section{Acknowledgements}

This project was supported by grants from the Ministry of Research and Development of Tunisia with participation of funds from the Ministry of Health of Tunisia.

\section{References}

[1] Guggenbuhll, P., Veillard, E., Quelvennec, E., Jego, P., Semana, G., Jean, S., Meadeb, J., Chalesl, G. and Perdriger, A. (2000) Analyse des Microsatellites a du TNF-Chez 35 Syndromes de Gougerot-Sjiigren Primitives. Revue Du Rhumatisme, 67, 362-367. http://dx.doi.org/10.1016/S1169-8330(00)80004-6

[2] Khani-Hanjani, A., Hoar, D., Horsman, D. and Keown, P. (2000) Identification of Four Novel Dinucleotide Repeat Polymorphisms in the TNF-a and TNF-b Genes. Human Immunology, 61, 511-512. 
http://dx.doi.org/10.1016/S0198-8859(99)00188-3

[3] http://www.ncbi.nlm.nih.gov/gv/mhc/xslcgi.cgi?cmd=mssearch

[4] Shi, Y.Y. and He, L. (2005) SHEsis, a Powerful Software Platform for Analyses of Linkage Disequilibrium, Haplotype Construction, and Genetic Association at Polymorphism Loci. Cell Research, 15, 97-98.

[5] Maalej, A., Hadj Kacem, H., Bellassoued, M., Abid, M., Makni, H. and Ayadi, H. (2000) Polymorphisms of HLA DQB1 CAR1/CAR2 and TNF $\alpha$ IR2/IR4 Microsatellite Markers in Patients Affected with Graves Disease. Clinical Immunology, 96, 91-93. http://dx.doi.org/10.1006/clim.2000.4881

[6] Crouau-Roy, B., Briant, L., Bouissou, C., Stavropoulos, C., Pociot, F., Thomsen, A.C. and Clayton, J. (1993) Tumor Necrosis Factor Microsatellites in Four European Populations. Human Immunology, 38, 213-216. http://dx.doi.org/10.1016/0198-8859(93)90543-A

[7] Törn, C., Hillman, M., Sanjeevi, C.B. and Olsson, M.L. (2006) Polymorphisms of TNF Microsatellite Marker A and HLA-DR-DQ in Diabetes Mellitus-A Study in 609 Swedish Subjects. Human Immunology, 67, 527-534. http://dx.doi.org/10.1016/j.humimm.2006.04.002

[8] Peñay, J.A., Calderony, R., Perez-Miranday, A., Vidalesy, C., Dugoujon, J.M., Carriony, M. and Crouau-Roy, B. (2002) Microsatellite DNA Markers from HLA Region (D6S105, D6S265 and TNFa) in Autochthonous Basques from Northern Navarre (Spain). Annals of Human Biology, 29, 176-191. http://dx.doi.org/10.1080/03014460110075675 